\title{
Measurement of effective electron mass in biaxial tensile strained silicon on insulator
}

S. F. Feste, Th. Schäpers, D. Buca, Q. T. Zhao, J. Knoch, M. Bouhassoune, Arno Schindlmayr, and S. Mantl

Citation: Appl. Phys. Lett. 95, 182101 (2009);

View online: https://doi.org/10.1063/1.3254330

View Table of Contents: http://aip.scitation.org/toc/apl/95/18

Published by the American Institute of Physics

\section{Articles you may be interested in}

Band structure, deformation potentials, and carrier mobility in strained $\mathrm{Si}, \mathrm{Ge}$, and $\mathrm{SiGe}$ alloys Journal of Applied Physics 80, 2234 (1996); 10.1063/1.363052

Six-band $\mathrm{k} \cdot \mathrm{p}$ calculation of the hole mobility in silicon inversion layers: Dependence on surface orientation, strain, and silicon thickness

Journal of Applied Physics 94, 1079 (2003); 10.1063/1.1585120

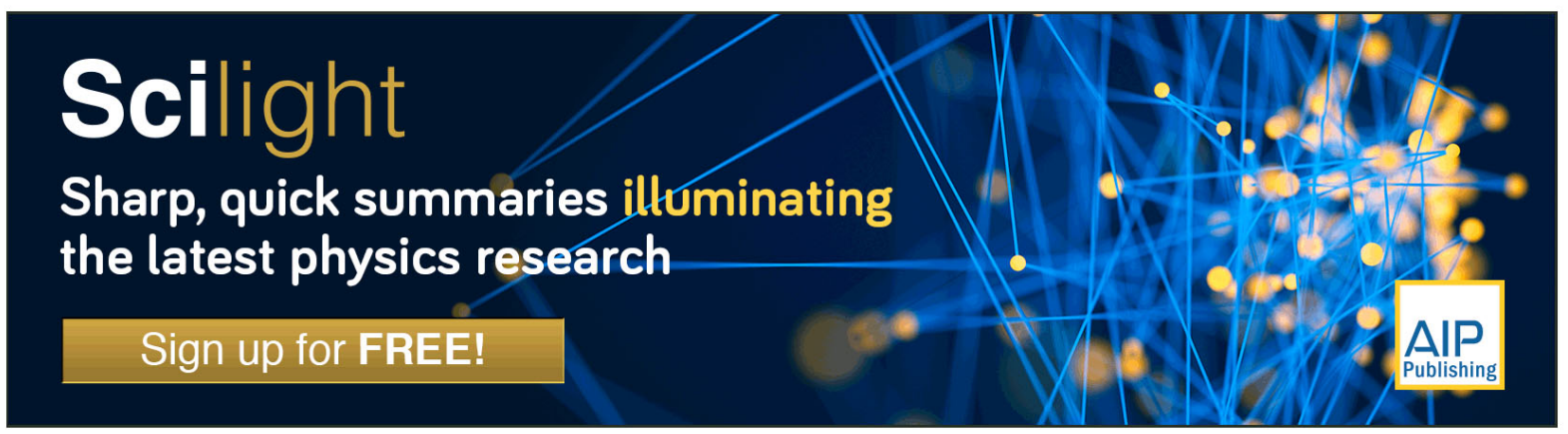




\title{
Measurement of effective electron mass in biaxial tensile strained silicon on insulator
}

\author{
S. F. Feste, ${ }^{1, a)}$ Th. Schäpers, ${ }^{1}$ D. Buca, ${ }^{1}$ Q. T. Zhao, ${ }^{1}$ J. Knoch, ${ }^{2}$ M. Bouhassoune,${ }^{3}$ \\ Arno Schindlmayr, ${ }^{3}$ and S. Mant $1^{1}$ \\ ${ }_{1}^{1}$ Institute of Bio- and Nanosystems (IBN1) and JARA-FIT Fundamentals of Future Information Technology, \\ Forschungszentrum Jülich, 52425 Jülich, Germany \\ ${ }^{2}$ Micro- and Nanoelectronics Devices Group, TU Dortmund University, 44227 Dortmund, Germany \\ ${ }^{3}$ Department Physik, Universität Paderborn, 33095 Paderborn, Germany
}

(Received 16 July 2009; accepted 6 October 2009; published online 2 November 2009)

\begin{abstract}
We present measurements of the effective electron mass in biaxial tensile strained silicon on insulator (SSOI) material with $1.2 \mathrm{GPa}$ stress and in unstrained SOI. Hall-bar metal oxide semiconductor field effect transistors on $60 \mathrm{~nm}$ SSOI and SOI were fabricated and Shubnikov-de Haas oscillations in the temperature range of $T=0.4-4 \mathrm{~K}$ for magnetic fields of $B=0-10 \mathrm{~T}$ were measured. The effective electron mass in SSOI and SOI samples was determined as $m_{\mathrm{t}}$ $=(0.20 \pm 0.01) m_{0}$. This result is in excellent agreement with first-principles calculations of the effective electron mass in the presence of strain. (C) 2009 American Institute of Physics.

[doi:10.1063/1.3254330]
\end{abstract}

Strain is employed in state-of-the art metal oxide semiconductor field effect transistors (MOSFETs) to enhance the intrinsic transport properties of silicon. Depending on the type of strain, electron and/or hole mobilities can be enhanced substantially, resulting in improved on-current, transconductance, etc., without geometrical scaling. ${ }^{1}$ These enhancements are attributed either to strain-induced subband splitting, band warping, or reduced scattering.

In this letter we show that the performance improvement of $n$-type FETs caused by biaxial tensile strain is due to preferential occupation of the lowest $\Delta_{2}$ subband with smaller conductivity mass $m_{\mathrm{t}}$ and a reduction in scattering. By measuring the conductivity mass we show that the curvature of the Si conduction band is not changed by biaxial tensile strain. This experimental result is confirmed by firstprinciples calculations of the effective electron mass under biaxial tensile strain.

Hall-bar MOSFETs $(L=670 \mu \mathrm{m}, W=140 \mu \mathrm{m})$ were fabricated on (001) $p$-doped silicon on insulator (SOI) and biaxial tensile strained SOI $(\mathrm{SSOI})\left(N_{\mathrm{A}}=1 \times 10^{15} \mathrm{~cm}^{-3}\right)$ with a thickness of $60 \mathrm{~nm}$ employing a standard MOSFET process. The SSOI was fabricated using our unique thinvirtual-substrate technology combined with wafer bonding and layer transfer. Details of the SSOI fabrication process can be found elsewhere. ${ }^{2}$ The stress in the SSOI layer was determined by Raman spectroscopy and Rutherford backscattering/channeling measurements to $\sigma_{\text {biax }}=1.2 \mathrm{GPa}$. The inset of Fig. 1 shows a sketch of the fabricated Hall-bar MOSFET.

Room-temperature electrical characterization of the devices showed good subthreshold slopes and $I_{\mathrm{on}} / I_{\text {off }}$ ratios. SSOI devices feature a factor 1.7 larger on-currents (Fig. 1) and a factor 1.8 larger transconductances (not shown) compared with devices on SOI. Electron mobility was measured employing a split capacitance-voltage $(\mathrm{C}-\mathrm{V})$ technique. Biaxial tensile strain improved the electron mobility by a factor of 1.7 compared with SOI (e.g., for a vertical electrical

${ }^{a)}$ Electronic mail: s.feste@fz-juelich.de. field of $E_{\mathrm{vert}}=2 \times 10^{5} \mathrm{~V} / \mathrm{cm}: \mu_{\mathrm{SOI}}=625 \mathrm{~cm}^{2} / \mathrm{V} \mathrm{s}, \quad \mu_{\mathrm{SSOI}}$ $=1060 \mathrm{~cm}^{2} / \mathrm{V} \mathrm{s}$ ). These improvements are in agreement with results reported by Takagi et al. $^{3}$ for the same strain states.

Magnetotransport measurements were performed in a ${ }^{3} \mathrm{He}$ cryostat in the temperature range of $T=0.4-4 \mathrm{~K}$ for magnetic fields of $B=0-10 \mathrm{~T}$ using a lock-in technique. Figure 2 shows Shubnikov-de Haas $(\mathrm{SdH})$ oscillations in the longitudinal resistance measured for a gate voltage of $V_{\mathrm{g}}$ $=1.6 \mathrm{~V}$ at different temperatures on the $60 \mathrm{~nm}$ SSOI sample. The inset shows the Fourier transform (FT) of the oscillations at $T=0.4 \mathrm{~K}$. A single peak in the FT confirms that only the lowest $\Delta_{2}$ subband is occupied. The frequency of the oscillations in the longitudinal resistance as a function of the inverse magnetic field is related to the electron concentration by $n_{\mathrm{s}}=g_{\mathrm{s}} g_{\mathrm{v}} e /[h \Delta(1 / B)]$, where $g_{\mathrm{s}}$ and $g_{\mathrm{v}}$ are the spin and valley degeneracies, $h$ is Planck's constant, $e$ the elementary charge, and $B$ the magnetic field. ${ }^{4}$ In Fig. 3(a) the peak positions in the reciprocal magnetic field of the $\mathrm{SdH}$ oscilla-

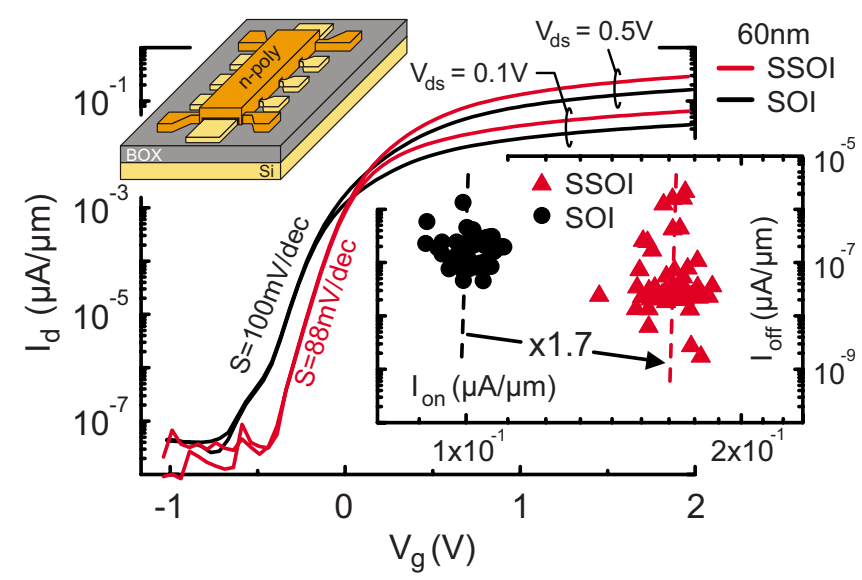

FIG. 1. (Color online) The left inset shows a sketch of the fabricated Hallbar MOSFETs on SSOI and SOI. The main panel displays the transfer characteristics of $n$-type Hall-bar MOSFETs on $60 \mathrm{~nm}$ biaxial tensile strained SSOI and SOI. The right inset shows a statistical $I_{\text {on }} / I_{\text {off }}$ plot of about 80 devices on each SSOI and SOI. 


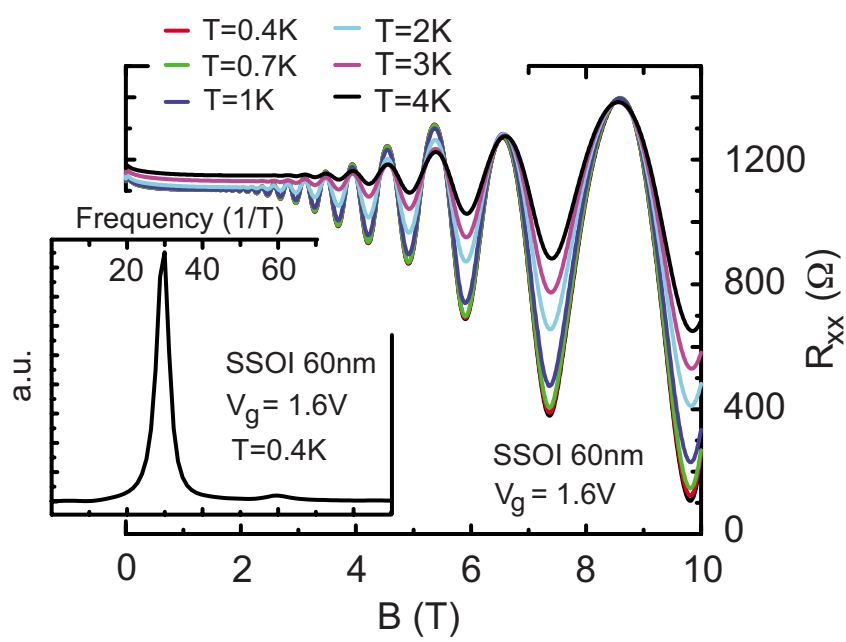

FIG. 2. (Color online) $\mathrm{SdH}$ oscillations in the longitudinal resistance $R_{x x}$ measured on SSOI Hall-bar MOSFET. The inset shows the FT of the oscillations featuring a single peak, confirming that only the lowest subband is occupied.

tions are plotted as a function of the peak number for three different gate voltages, corresponding to three different electron concentrations. The electron concentrations were determined from the slopes of the linear fits to the peak positions in the reciprocal magnetic field as $n_{\mathrm{s}}=0.95,2.06$, and 2.88 $\times 10^{12} \mathrm{~cm}^{-2}$.

The effective electron mass was extracted from the temperature dependence of the $\mathrm{SdH}$ oscillation amplitudes $A$ at a fixed magnetic field $B$. After subtraction of the nonoscillatory background the natural logarithm of the oscillation amplitude $A$ divided by the temperature $T$ is plotted as a function of the temperature [inset of Fig. 3(b)]. The data were fitted using a nonlinear least-squares technique with the effective electron mass $m_{\text {eff }}$ and a constant $C$ as parameters ${ }^{5}$

$$
\ln \left(\frac{A}{T}\right)=C-\ln \left[\sinh \left(\frac{2 \pi^{2} k_{\mathrm{B}} T m_{\mathrm{eff}}}{e \hbar B}\right)\right],
$$

where $k_{\mathrm{B}}$ is Boltzmann's constant.

The extracted effective electron mass of $m_{\text {eff }}$ $=(0.20 \pm 0.01) m_{0}$ in SSOI and SOI devices is in excellent agreement with the transverse electron mass $m_{\mathrm{t}}$ of electrons in the $\Delta_{2}$ subband and with values for $m_{\text {eff }}$ reported for unstrained bulk Si MOSFETs by other authors. ${ }^{6-8}$ This demonstrates experimentally that biaxial tensile stress of about 1.2 GPa does not warp the $\Delta_{2}$ conduction-band constant-energy surfaces for in-plane directions. The electron mobility en-
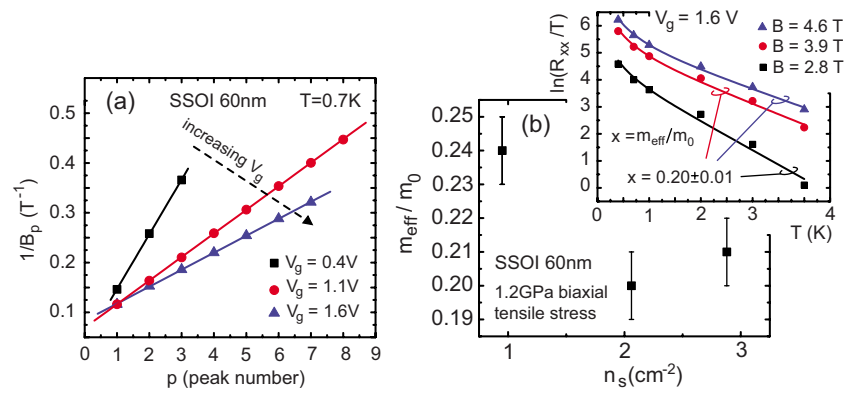

FIG. 3. (Color online) (a) Electron concentration determined from fits to the peak positions in the reciprocal magnetic field. (b) The inset shows the fits for the effective electron mass. The main panel displays the dependence of the effective electron mass on the carrier concentration.
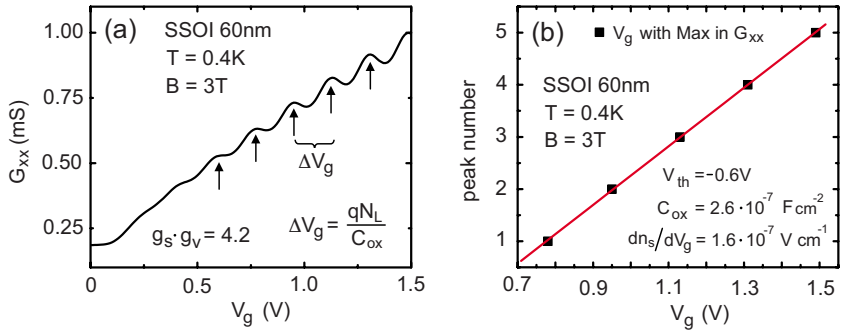

FIG. 4. (Color online) (a) Conductance as a function of the gate voltage. (b) Gate voltages with peak values of the conductance plotted vs gate voltage. Threshold voltage, gate capacitance and $d n_{\mathrm{s}} / d V_{\mathrm{g}}$ can be deduced from the linear fit.

hancement observed in $n$-FETs on biaxial tensile strained SOI is, therefore, caused by the larger subband splitting due to the additive contributions of confinement and strain $(\Delta E$ $>200 \mathrm{meV}$ ). This has two effects: (i) electrons occupy only the lowest $\Delta_{2}$ subband at room temperature with the small mass $m_{\mathrm{t}}$ in transport direction, while in SOI a fraction of electrons occupies the $\Delta_{4}$ valleys. The mobility enhancement due to mass change alone accounts for a mobility improvement of about 1.3; (ii) lifting of the valley degeneracy and the large energy splitting reduces intervalley phonon scattering effectively and accounts for the additional mobility enhancement. In the main panel of Fig. 3(b) the effective electron mass in biaxial tensile strained $\mathrm{Si}$ is plotted versus the electron concentration. At a low carrier concentration of $n_{\mathrm{s}}$ $\approx 1 \times 10^{12} \mathrm{~cm}^{-2}$ an increase in the effective mass is observed. The measured value of $m_{\mathrm{eff}}=0.24 m_{0}$ is larger than reported by Smith and Stiles ${ }^{9}$ but in good agreement with more recent results reported by Pan et al. ${ }^{6}$ and by Dragosavac et al. ${ }^{8}$

Figure 4(a) shows the conductance as a function of the gate voltage for a constant magnetic field of $B=3 \mathrm{~T}$. In contrast to the usual $\mathrm{SdH}$ experiment, which measures changes in conductance as the Landau levels move through the Fermi surface, here the Fermi surface moves through the Landau levels. ${ }^{10}$ Because the period of the conductance oscillations is constant, the measurement indicates that each Landau level contains the same number of states as given by ${ }^{4} N_{\mathrm{L}}$ $=g_{\mathrm{s}} g_{\mathrm{v}} e B / h$. The period of the oscillations equals the change in $V_{\mathrm{g}}$, which leads to a change in $N_{\mathrm{L}}$ in the electron concentration $n_{\mathrm{s}}$. Therefore, the maxima in the conductance occur when the gate voltage satisfies

$$
V_{\mathrm{g}}=i\left(\frac{g_{\mathrm{s}} g_{\mathrm{v}} e^{2}}{h}\right) B+V_{\mathrm{th}},
$$

where $i$ is an integer and $V_{\text {th }}$ the threshold voltage. Figure 4(b) shows a plot of the number of the conductance maxima against the gate voltages at which they occur. From the slope of the linear fit the change in carrier concentration with gate voltage was found to be $d n_{\mathrm{s}} / d V_{\mathrm{g}}=1.69 \times 10^{7} \mathrm{~V}^{-1} \mathrm{~cm}^{-2}$. The intercept with the voltage axis yields a threshold voltage of $V_{\text {th }}=-0.6 \mathrm{~V}$. Knowing the carrier density as a function of the gate voltage, the product of valley and spin degeneracy $g_{\mathrm{v}} g_{\mathrm{s}}$ can be determined from the period of the conductance oscillations. It was found to be 4.2 in satisfactory agreement with the expected value of 4 for the $\Delta_{2}$ subband of the $\mathrm{Si}$ conduction band and spin degeneracy. The capacitance of the Hall-bar MOSFET was determined as $C_{\mathrm{ox}}=2.6$ $\times 10^{-7} \mathrm{~F} \mathrm{~cm}^{-2}$ in good agreement with $\mathrm{C}-\mathrm{V}$ measurements. 

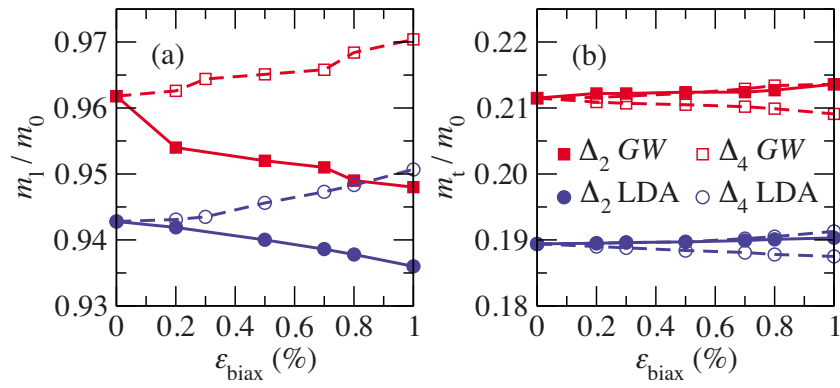

FIG. 5. (Color online) Longitudinal (a) and transversal (b) effective electron mass for the $\Delta_{2}$ and $\Delta_{4}$ valleys as a function of biaxial tensile strain, obtained from the conduction-band curvature calculated in the LDA or the $G W$ approximation.

To support our experimental findings the effective electron mass was studied theoretically from first principles. The lattice geometry of strained $\mathrm{Si}$ was determined using densityfunctional theory ${ }^{11}$ in the local-density approximation (LDA). ${ }^{12}$ A nonlocal norm-conserving pseudopotentials, a plane-wave basis set with a cutoff energy of $20 \mathrm{Ry}$, and 10 $\times 10 \times 10$ mesh points in the full Brillouin zone were used. The temperature was set to $T=0 \mathrm{~K}$ since it has negligible effect on the band dispersion. For tensile strain in the (001) plane we find a Poisson ratio of 0.79 describing the volume relaxation in the growth direction. As the Kohn-Sham eigenvalues are known to differ systematically from photoemission data, we apply a perturbative self-energy correction within the $G W$ approximation ${ }^{13}$ to arrive at a more accurate quasiparticle band structure. The self-energy is constructed with $4 \times 4 \times 4$ mesh points and 100 unoccupied bands, ${ }^{14}$ yielding the correct indirect band gap of $1.17 \mathrm{eV}$ for unstrained Si compared with $0.46 \mathrm{eV}$ in the LDA. For a strain of $\varepsilon_{\text {biax }}=0.65 \%$ that corresponds to the measured stress of $1.2 \mathrm{GPa}$ we find a split of $0.10 \mathrm{eV}$ between the $\Delta_{2}$ and $\Delta_{4}$ valleys and an overall reduction of the band gap by $0.12 \mathrm{eV}$. The effective electron mass is deduced from the curvature of polynomial fits to the quasiparticle energies at the conduction-band minima. The results in Fig. 5 confirm the very weak dependence of all elements of the effective-mass tensors on the strain. Relative to the LDA, which is included here for comparison with earlier calculations at this level of theory, ${ }^{15}$ the $G W$ approximation predicts systematically larger effective masses. The increase is due to the positively charged exchange-correlation hole around an electron, which acts as a quantum well offering additional resistance to mobility, and mathematically linked to the redistribution of spectral weight from the main quasiparticle peak to satellite resonances. ${ }^{16}$ For the transverse component of the $\Delta_{2}$ valleys at $\varepsilon_{\text {biax }}=0.65 \%$ the extracted $m_{\mathrm{t}}=0.21 m_{0}$ is in excellent agreement with the experimental measurements.

In conclusion, we have measured the in plane effective electron mass in biaxial tensile strained SOI with a stress of 1.2 GPa and in unstrained SOI. For both materials the effective mass in the transport direction was found to be $m_{\mathrm{t}}$ $=(0.20 \pm 0.01) m_{0}$ in perfect agreement with effective-mass values calculated from first-principles theory in the $G W$ approximation. The measured improvements through strain by a factor of $\approx 1.7$ at room temperature in $\mu, I_{\mathrm{on}}$, and $g_{\mathrm{m}}$ are therefore caused by the larger subband splitting in SSOI due to the additive contributions of confinement and strain. This leads to a smaller conductivity mass in SSOI and reduced intersubband scattering compared with SOI.

This work received funding from the EU through the NANOSIL Network of Excellence (Contract No. ICT-2007216171) and the European Theoretical Spectroscopy Facility e-I3 (Contract No. INFRA-2007-211956), and from the German Federal Ministry of Education and Research via the MEDEA+ project DECISIF (2T104).

${ }^{1}$ Y. Sun, S. E. Thompson, and T. Nishida, J. Appl. Phys. 101, 104503 (2007).

${ }^{2}$ D. Buca, B. Holländer, H. Trinkaus, S. Mantl, R. Carius, R. Loo, M. Caymax, and H. Schaefer, Appl. Phys. Lett. 85, 2499 (2004).

${ }^{3}$ S. Takagi, T. Mizuno, T. Tezuka, N. Sugiyama, S. Nakaharai, T. Numata, J. Koga, and K. Uchida, Solid-State Electron. 49, 684 (2005).

${ }^{4}$ T. Ando, A. B. Fowler, and F. Stern, Rev. Mod. Phys. 54, 437 (1982).

${ }^{5}$ A. Saxler, P. Debray, R. Perrin, S. Elhamri, W. C. Mitchel, C. R. Elsass, I. P. Smorchkova, B. Heying, E. Haus, P. Fini, J. P. Ibbetson, S. Keller, P. M Petroff, S. P. DenBaars, U. K. Mishra, and J. S. Speck, J. Appl. Phys. 87, 369 (2000).

${ }^{6}$ W. Pan, D. C. Tsui, and B. L. Draper, Phys. Rev. B 59, 10208 (1999).

${ }^{7}$ F. F. Fang, A. B. Fowler, and A. Hartstein, Phys. Rev. B 16, 4446 (1977).

${ }^{8}$ M. Dragosavac, D. J. Paul, M. Pepper, A. B. Fowler, and D. A. Buchanan, Semicond. Sci. Technol. 20, 664 (2005).

${ }^{9}$ J. L. Smith and P. J. Stiles, Phys. Rev. Lett. 29, 102 (1972).

${ }^{10}$ A. B. Fowler, F. F. Fang, W. E. Howard, and P. J. Stiles, Phys. Rev. Lett. 16, 901 (1966)

${ }^{11}$ P. Hohenberg and W. Kohn, Phys. Rev. 136, B864 (1964).

${ }^{12}$ W. Kohn and L. J. Sham, Phys. Rev. 140, A1133 (1965).

${ }^{13}$ L. Hedin, Phys. Rev. 139, A796 (1965).

${ }^{14}$ M. M. Rieger, L. Steinbeck, I. D. White, H. N. Rojas, and R. W. Godby, Comput. Phys. Commun. 117, 211 (1999); C. Freysoldt, P. Eggert, P. Rinke, A. Schindlmayr, R. W. Godby, and M. Scheffler, ibid. 176, 1 (2007).

${ }^{15}$ D. Yu, Y. Zhang, and F. Liu, Phys. Rev. B 78, 245204 (2008).

${ }^{16}$ M. Oshikiri, F. Aryasetiawan, Y. Imanaka, and G. Kido, Phys. Rev. B 66, 125204 (2002). 